\title{
Comparações entre famílias de meio irmãos paterno através do fator de condição de Fulton, alométrico e a taxa de crescimento em curimbatá (Prochilodus lineatus)
}

\author{
Comparisons among paternal half-sib families by fulton and \\ alometric condition factors and growth rate in curimbatá \\ (Prochilodus lineatus)
}

\author{
Marco Antônio da Rocha ${ }^{1 *}$; Edson Luis de Azambuja Ribeiro'; Ivone Yurika \\ Mizubuti ${ }^{1}$; Julian Cristina Borosky²; Mariana Isa Poci Palumbo Antunes ${ }^{3}$
}

\section{Resumo}

A determinação do fator de condição em peixes tem sido feita para informar o crescimento, reprodução e sobrevivência em um determinado ambiente. Com o objetivo de comparar grupos de meio-irmãos paterno, foi realizado um experimento na Estação de Piscicultura da Universidade Estadual de Londrina, em uma amostra constituida de 253 animais, filhos de 10 reprodutores curimbatá (Prochilodus lineatus). Foi determinado o cálculo do fator de condição de Fulton, alométrico e a taxa de crescimento para verificar a possibilidade de seleção para essas características. Os resultados indicam que a maioria dos reprodutores apresentam crescimento alométrico negativo, representado por $b<3,0$, a exceção de um reprodutor cuja progênie apresentou crescimento isométrico $(b=3,0)$. A identificação dos reprodutores cujas progênies tiveram maior taxa de crescimento foi através do fator de condição alométrico. $O$ fator de condição de Fulton que utiliza para o cálculo o coeficiente de regressão de $b=3,0$ não se mostrou viável. A seleção para a melhora das características fator de condição alométrico e taxa de crescimento é possível com a utilização da seleção pela progênie, pois a herdabilidade para essas características é baixa $\left(h^{2}=0,20\right)$.

Palavras-chave: Fator de condição, peixe, carpa

\begin{abstract}
The determination of the condition factor in fish has been done to inform the growth, reproduction and survival in a specific environment. With the objective of comparing groups of paternal half-sibs it was conducted an experiment at the Fish Station of the State University of Londrina. It was used a sample of 253 fishes, sons of 10 Curimbata (Prochilodus lineatus) reproducers. In order to verify the possibility of their use in selection, Fulton and alometric condition factors were determined, as well as the growth rate. The results suggested that the progeny of the majority of the reproducers presented negative alometric growth $(b<3.0)$. There was only one exception, where the progeny of one male showed an isometric growth $(b=3.0)$. The identification of reproducers, which the progeny presented greater growth rate, was done by the alometric condition factor. The condition factor of Fulton, which uses the regression coefficient of $b=3.0$, was not viable. Selection to improve the traits alometric condition factor and growth rate is possible with the utilization of the progeny selection, since these traits present low heritability $\left(\mathrm{h}^{2}=0.20\right)$.
\end{abstract}

Key words: Condition factor, fish, carp

1 Professores associados do departamento de Zootecnia da Universidade Estadual de Londrina, Londrina, Paraná, Brasil. E-mail: marco@uel.br

2 Médica Veterinária

3 Acadêmica do Curso de Medicina Veterinária da Universidade Estadual de Londrina, Londrina, Paraná, Brasil.

* Autor para correspondência 


\section{Introdução}

A utilização do peixe, como alimento, tem sido incrementado em todo o mundo. Na Ásia, esse procedimento tem sido alvo de pesquisas há muito tempo, uma vez que constitui uma das fontes mais importantes de proteína animal. No Brasil, a criação de peixes tem tido ultimamente uma atenção maior por parte de órgãos governamentais e iniciativa privada no sentido de proporcionar à população, sempre crescente, uma oferta de fonte alimentar protéica mais acessível e com menores custos.

$\mathrm{Na}$ criação dos peixes, a determinação da condição dos indivíduos tem sido feita para informar os vários aspéctos da população a que pertence. A exposição a substâncias químicas, nutrição, doenças e contaminantes interferem na condição dos peixes e pode variar razoavelmente de local para local dentro da mesma população (DOYON; DOWNING; MANIN, 1988; FISHER; WILLIS; POPE, 1996).

Adeterminação dacondição serve como indicativo da reserva de energia, com a expectativa que um peixe tem em demonstrar altas taxas de crescimento, o potencial reprodutivo e a sobrevivência naquele ambiente. Pode ser feito através de medidas morfométricas (peso e comprimento), fisiológico (pesos do fígado e gônadas) e valores bioquímicos (lipídios e proteínas), conforme Lloret et al. (2002).

A estimativa do fator de condição, através da morfometria, é determinada pela relação entre peso corporal e comprimento corporal total, com a transformação de ambos para logarítimo natural e posterior determinação do intercepto e o coeficiente de regressão, que pode variar de 2,5 a 4,0 (isométrico é igual a 3,0), descreve a curva da relação, segundo Royce (1972). A estimativa do fator de condição pode ser feita de três formas: Fulton, alométrico e o relativo ou de Le Cren, conforme Le Cren (1951).

O fator de condição de Fulton assume que o crescimento é isométrico $(b=3,0)$, sendo determinado pela relação: peso/(comprimento corporal) $)^{3}$. O fator de crescimento alométrico parte do princípio que nem todo crescimento é isométrico $(b=3,0)$ e, dependendo de cada população, o valor de $b$ pode estar dentro da faixa de valores entre 2,5 a 4,0 .

O fator de condição relativo ao de Le Cren, de acordo com Le Cren (1951), foi desenvolvido para resolver deficiências no valor do fator de condição de Fulton $(b=3,0)$. É determinado pela relação entre o peso atual do animal dividido pelo seu peso predito. O objetivo deste trabalho foi comparar famílias de meios irmãos paternos através do fator de condição de Fulton, alométrico e a taxa de crescimento.

\section{Material e Métodos}

O experimento foi realizado na Estação de Piscicultura da Universidade Estadual de Londrina, PR. A localização da estação é $23^{\circ} 23^{\prime}$ de latitude sul, e $51^{\circ} 11^{\prime}$ de latitude oeste, com altitude média de 566 metros. O clima, segundo a classificação de Köppen, é do tipo cfa, ou seja, sub-tropical úmido.

A classificação do solo no local do experimento, de acordo com Rocha, Barros e Guimarães (1991), é terra roxa estruturada latossolisada eutrófica moderada, com textura argilosa. A amostra foi constituída de 253 peixes, filhos de 10 reprodutores curimbatá (Prochilodus lineatus).

Os animais foram criados até a idade de 150 dias em 10 tanques redes com dimensão de 2,25 $\mathrm{m}^{2}$ de superfície e altura de 1,2 metros, com fonte de oxigênio de tanque rede, sendo posteriormente marcados (conforme a família) utilizando o método de Welch e Mills (1981) e criados em três viveiros de $100 \mathrm{~m}^{2}$ de superfície com profundidade de 1,2 metros.

Além da alimentação natural, presente nos viveiros, iniciou-se a alimentação com uma ração contendo $40 \%$ de proteína bruta e $2900 \mathrm{Kcal}$ de energia metabolizável e representada por pequenas partículas microprotegidas para possibilitar a ingestão pelas larvas, conforme Kanazawa e Teshima (1988). Da fase de alevinagem até o final do experimento os animais receberam uma ração 
peletizada na quantidade de 3\% da biomassa, com $30 \%$ de proteína bruta e 2800 Kcal de energia metabolizável.

A qualidade da água foi determinada a cada 15 dias, sendo as seguinte: temperatura, ph, condutividade elétrica e o oxigênio dissolvido, a partir da transferência dos animais dos tanques redes para os viveiros.

As características peso corporal e comprimento corporal foram determinadas em idades entre 60 e 330 dias. A relação foi determinada para verificar o tipo de crescimento (isométrico ou alométrico), aplicando o " t" teste, conforme Sokal e Rohlf(1980), para os grupos de meio irmãos paternos e geral. $\mathrm{O}$ cálculo do intercepto e do coeficiente de regressão foram determinados através da transformação das variáveis peso corporal e comprimento corporal total para logarítimo natural.

O fator de condição de Fulton foi determinado conforme fórmula apresentada por Frank e Fowler (2001):

$$
\mathrm{K}_{\mathrm{F}}=\left(\mathrm{W} / \mathrm{L}^{3}\right) \times 100
$$

Sendo que:

$\mathrm{K}_{\mathrm{F}}=$ Fator de condição de Fulton,

$\mathrm{W}=$ Peso corporal em gramas,

$\mathrm{L}=$ Comprimento corporal total em centímetros.

O fator de condição alométrico foi determinado conforme fórmula presentada por Le Cren (1951):

$$
\mathrm{K}_{\mathrm{A}}=\left(\mathrm{W} / \mathrm{L}^{\mathrm{b}}\right) \times 100
$$

Sendo que:

$\mathrm{K}_{\mathrm{A}}=$ Fator de condição alométrico,

$\mathrm{b}=$ Coeficiente de regressão.
A taxa de crescimento instantânea (igr (\%)) foi determinada através de fórmula apresentada por Ricker (1979):

$$
\operatorname{igr}(\%)=\left(\mathrm{L}_{\mathrm{n}} \mathrm{W}_{\mathrm{f}}-\mathrm{L}_{\mathrm{n}} \mathrm{W}_{\mathrm{i}}\right) /\left(\mathrm{t}_{\mathrm{f}}-\mathrm{t}_{\mathrm{i}}\right)
$$

Sendo que:

Igr $(\%)=$ Taxa de crescimento instantânea,

$\mathrm{L}_{\mathrm{n}} \mathrm{W}_{\mathrm{f}}=$ Logarítimo natural do peso final,

$\mathrm{L}_{\mathrm{n}} \mathrm{W}_{\mathrm{i}}=$ Logarítimo natural do peso inicial,

$\mathrm{t}_{\mathrm{f}}=$ Idade final,

$\mathrm{t}_{\mathrm{i}}=$ Idade inicial.

Os dados foram analisados, utilizando o Programa SAEG (UNIVERSIDADE FEDERAL DE VIÇOSA, 1983), conforme o modelo:

$$
\mathrm{Y}_{\mathrm{ijk}}=\mu+\mathrm{I}_{\mathrm{i}}+\mathrm{R}_{\mathrm{j}}+\mathrm{IR}_{\mathrm{ij}}+\mathrm{C}_{\mathrm{ijk}}
$$

Sendo que:

$\mathrm{Y}_{\mathrm{ijk}}=$ Fator de condição de Fulton ou alométrico dos peixes de idade i-ésima do reprodutor j-ésimo,

$\mu=$ Média geral,

$\mathrm{I}_{\mathrm{i}}=$ Efeito da idade i-ésima,

$\mathrm{R}_{\mathrm{j}}=$ Efeito do reprodutor $\mathrm{j}$-ésimo,

$\mathrm{IR}_{\mathrm{ij}}=$ Efeito da interação da idade i-ésima com o reprodutor j-ésimo,

$\mathrm{C}_{\mathrm{ijk}}=$ Erro experimental.

As médias foram comparadas através do teste de Tukey ao nível de 5\% de probabilidade.

\section{Resultados e Discussão}

$\mathrm{Na}$ tabela 1 são apresentados os resultados obtidos na avaliação da qualidade da água. 
Tabela 1. Número de determinações (N), média, desvio padrão (s) e coeficiente de variação (CV (\%)) das características da qualidade da água obtidas nos viveiros externos, durante o experimento.

\begin{tabular}{ccccc}
\hline Característica & N & Média & s & CV (\%) \\
\hline Temperatura $\left({ }^{\circ} \mathrm{C}\right)$ & 12 & 22,46 & 1,82 & 8,10 \\
$\mathrm{pH}$ & 12 & 6,99 & 0,58 & 8,30 \\
Condutividade elétrica $\left(\mu \mathrm{scm}^{-1}\right)$ & 12 & 56,98 & 5,18 & 9,09 \\
Oxigênio dissolvido $\left(\mathrm{mg} \cdot .^{-1}\right)$ & 8 & 6,90 & 0,49 & 7,10 \\
\hline
\end{tabular}

Os valores da tabela estão de acordo com os citados por Padua (1994), indicando que durante o experimento a qualidade da água manteve dentro dos parâmetros normais recomendados. A relação encontrada entre peso corporal e comprimento corporal total é potencial, conforme o gráfico 1 .

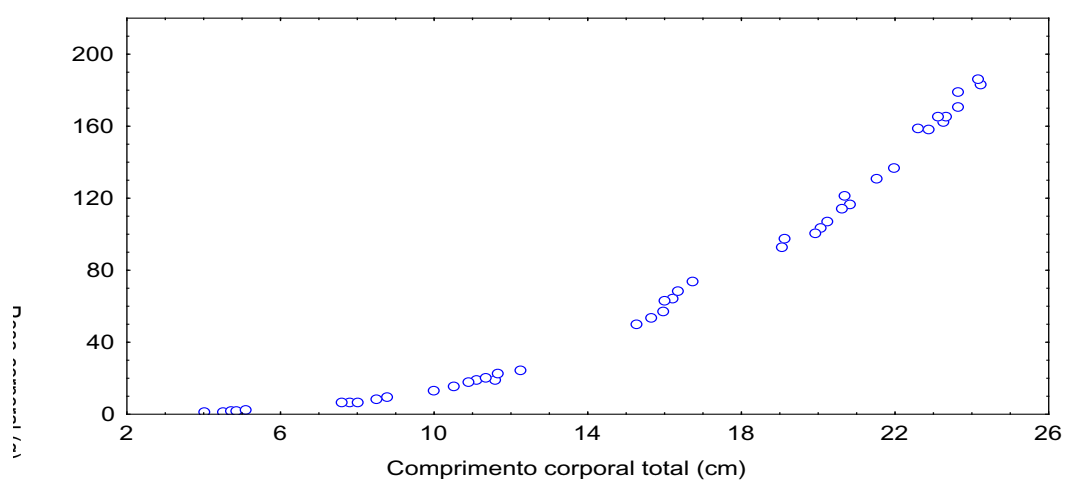

Gráfico 1. Relação entre o peso e o comprimento corporal dos peixes (Prochilodus lineatus).

Isto indica que no período inicial os peixes se desenvolvem mais no sentido do comprimento corporal total, e após o peso mostra um incremento maior que o comprimento corporal total, o que concorda com Ricker (1979), Rocha (1995) e Rocha et al. (2000).

Os valores da relação peso-comprimento e tipo de crescimento de acordo com as famílias de meiosirmãos paternos são apresentados na tabela 2 .

$\mathrm{O}$ valor determinado, considerando a amostra total $(b=2,90)$, indica que está dentro da faixa de valores apresentado por Le Cren (1951) e Royce (1972). Comparando esse valor com o encontrado no curimbatá por Mainardes Pinto et al. (1984), verifica-se que é praticamente o mesmo valor de b. Por outro lado, é um valor muito próximo aos encontrados por Gunnes e Gjedrem (1981), Gjerde e Schaeffer (1989) na truta arco-íris, Refstie e Steine (1978) no salmão, Leite et al. (1984) e Toledo Filho e Santos (1987) apud Galhardo (1989) no curimbatá.

Observa-se também nesta tabela que o valor do coeficiente de regressão (b) e o intercepto (lna) estão associados negativamente, isto é, valores maiores de $\mathrm{B}$ indicam valores menores de lna e vice-versa. Nesta relação, todas as famílias de meios-irmãos paternos apresentam alometria negativa $(p<0,05)$. A única exceção é o reprodutor $n^{\circ} 40$, cujos filhos apresentaram isometria $(\mathrm{p}>0,05)$ para a relação.

A idade dos animais apresentam diferenças significativas $(p<0,05)$ para o fator de condição de Fulton. Na tabela 3 são apresentados os valores médios do fator de condição de Fulton e alométrico, conforme a idade. 
Tabela 2. Valores da relação peso $\mathrm{x}$ comprimento, intercepto (lna), coeficiente de determinação $\left(\mathrm{r}^{2}\right)$ e tipo de crescimento, conforme a família de meios-irmãos paternos.

\begin{tabular}{cccccc}
\hline $\mathbf{N}^{\mathbf{0}}$ do reprodutor & $\mathbf{N}$ & $\mathbf{I n a}$ & $\mathbf{b}$ & $\mathbf{r}^{\mathbf{2}}$ & Tipo de crescimento \\
\hline 10 & 42 & 0,01610 & 2,93 & 0,99 & Alo \\
20 & 24 & 0,02007 & 2,83 & 0,96 & Alo \\
30 & 16 & 0,01816 & 2,88 & 0,97 & Alo \\
40 & 25 & 0,01436 & 2,97 & 0,99 & Iso \\
50 & 40 & 0,01567 & 2,95 & 0,99 & Alo \\
60 & 15 & 0,02033 & 2,86 & 0,99 & Alo \\
70 & 27 & 0,02605 & 2,83 & 0,95 & Alo \\
80 & 22 & 0,01812 & 2,89 & 0,99 & Alo \\
90 & 30 & 0,01559 & 2,95 & 0,99 & Alo \\
100 & 12 & 0,02016 & 2,87 & 0,99 & Alo \\
\hline Geral & 253 & 0,01738 & 2,90 & 0,98 & Alo
\end{tabular}

* Tipo de crescimento (Alo = alométrico e Iso = isométrico) através do "t" teste ao nível de 5 .

Tabela 3. Valores médios do fator de condição de Fulton $\left(\mathrm{F}_{\mathrm{F}}\right)$ e alométrico $\left(\mathrm{F}_{\mathrm{A}}\right)$, conforme a idade em dias.

\begin{tabular}{ccccc}
\hline Idade (dias) & $\mathbf{F}_{\mathbf{F}}$ & $\mathbf{C V}(\mathbf{\%})$ & $\mathbf{F}_{\mathbf{A}}$ & $\mathbf{C V}(\mathbf{\%})$ \\
\hline 60 & $1,5307 \mathrm{a}$ & 13,0 & $1,8535 \mathrm{a}, \mathrm{b}$ & 16,7 \\
90 & $1,3705 \mathrm{~b}, \mathrm{c}$ & 4,1 & $1,7658 \mathrm{~b}$ & 15,0 \\
150 & $1,3046 \mathrm{c}$ & 5,6 & $1,7210 \mathrm{~b}$ & 18,4 \\
210 & $1,4613 \mathrm{a}, \mathrm{b}$ & 5,9 & $2,0222 \mathrm{a}$ & 18,8 \\
270 & $1,3083 \mathrm{c}$ & 3,4 & $1,8888 \mathrm{a}, \mathrm{b}$ & 19,0 \\
330 & $1,3049 \mathrm{c}$ & 3,5 & $1,8976 \mathrm{a}, \mathrm{b}$ & 17,0 \\
\hline
\end{tabular}

* Médias seguidas de mesma letra, nas colunas, não diferem estatisticamente pelo teste de Tukey (p>0,05).

O fator de condição alométrico e o fator de condição de Fulton apresentaram um maior valor na idade de 210 dias. Isto se prende ao fato de que tiveram melhores condições após a transferência dos animais que estavam nos tanques rede para os viveiros externos. Desta forma, com uma menor densidade, permitiu que tivesse um desenvolvimento maior.

Após essa idade e com o desenvolvimento dos animais, o fator de condição de Fulton diminuiu em relação à idade de 210 dias $(\mathrm{p}<0,05)$, e o fator de condição alométrico não apresentou diferença significativa $(\mathrm{p}>0,05)$. Por outro lado, a não coincidência das diferenças entre o fator de condição alométrico e o fator de condição de Fulton em idades entre 60 a 150 dias, indica que o fator de condição alométrico é mais sensível na identificação do desenvolvimento dos animais em relação à idade. Estas diferenças devem ser devido ao coeficiente de regressão, que no fator de condição de Fulton considera que qualquer relação entre peso corporal e comprimento corporal total em peixe, é isométrico, que pelos resultados obtidos não é verdadeiro.

$\mathrm{Na}$ tabela 4, são apresentados os valores médios do fator de condição de Fulton e alométrico, conforme as famílias de meios-irmãos paternos. 
Tabela 4. Médias e coeficiente de variação do fator de condição de Fulton $\left(\mathrm{F}_{\mathrm{F}}\right)$ e alométrico $\left(\mathrm{F}_{\mathrm{A}}\right)$, conforme as famílias de meios-irmãos paternos.

\begin{tabular}{ccccc}
\hline $\mathbf{N}^{\mathbf{0}}$ do reprodutor & $\mathbf{F}_{\mathbf{F}}$ & $\mathbf{C V} \mathbf{( \% )}$ & $\mathbf{F}_{\mathbf{A}}$ & $\mathbf{C V ~ ( \% )}$ \\
\hline 10 & $1,3503 \mathrm{a}$ & 5,2 & $1,6070 \mathrm{c}, \mathrm{d}$ & 3,0 \\
20 & $1,3605 \mathrm{a}$ & 9,6 & $2,0565 \mathrm{~b}$ & 7,8 \\
30 & $1,3328 \mathrm{a}$ & 7,1 & $1,8108 \mathrm{~b}, \mathrm{c}$ & 8,6 \\
40 & $1,3238 \mathrm{a}$ & 6,8 & $1,4275 \mathrm{~d}$ & 6,8 \\
50 & $1,3968 \mathrm{a}$ & 7,4 & $1,5820 \mathrm{c}, \mathrm{d}$ & 7,8 \\
60 & $1,4765 \mathrm{a}$ & 10,4 & $2,0680 \mathrm{~b}$ & 10,5 \\
70 & $1,3210 \mathrm{a}$ & 4,8 & $2,6027 \mathrm{a}$ & 11,8 \\
80 & $1,3770 \mathrm{a}$ & 6,9 & $1,8170 \mathrm{~b}, \mathrm{c}$ & 3,3 \\
90 & $1,4047 \mathrm{a}$ & 7,3 & $1,5900 \mathrm{c}, \mathrm{d}$ & 7,0 \\
100 & $1,4570 \mathrm{a}$ & 9,7 & $2,0200 \mathrm{~b}$ & 6,6 \\
\hline
\end{tabular}

* Médias seguidas de mesma letra, nas colunas, não diferem estatisticamente pelo teste de Tukey (p>0,05).

Os resultados indicam que as famílias de meios-irmãos paternos estão em boas condições de criação, sendo todos os valores superiores a 1 . No entanto, o fator de condição alométrico foi o que evidenciou melhor o desenvolvimento das famílias. $\mathrm{O}$ reprodutor $\mathrm{n}^{0} 70$, que com o fator de condição de Fulton se mostrou inferior à grande maioria dos outros reprodutores, no fator de condição alométrico apresentou superioridade a todos os outros, indicando que o desenvolvimento da sua progênie foi bem mais acentuado que a progênie dos outros reprodutores. Isto provavelmente tenha uma explicação em relação ao real valor do coeficiente de regressão que, ao contrário do fator de condição de Fulton, é variável conforme a amostra.

Os fatores que determinam a condição dos peixes podem ser, conforme Royce (1972): intrínsecos (genético e hormonal) e extrínsecos (densidade, clima, imunológicos, sanidade e, principalmente, a quantidade e a qualidade dos alimentos).

A herdabilidade para o fator de condição gira ao redor de 0,20, conforme Rocha et al. (2000) no curimbatá (Prochilodus lineatus), Mckay, Ihssen e Friars (1986); Gall e Huang (1988); Kinghorn (1983); Refstie (1980) na truta arco-íris (Salmo gairdneri); Beacham e Murray (1988) no salmão (Oncorhynchus gorbusha). Este valor indica que a seleção individual pode proporcionar pouco progresso genético. No entanto, em se tratando de informações de progênie (meios-irmãos paternos), a seleção para o reprodutor pode ser vantajoso com a obtenção de um progresso genético maior, uma vez que este tipo de seleção favorece as características de baixa herdabilidade, conforme Kirpichnikov (1981). Na tabela 5, são apresentados os valores da taxa de crescimento instantâneo (igr (\%)), considerando as famílias de meios-irmãos paternos.

De uma forma geral, com o aumento da idade verificou-se que o igr (\%) diminui $(r=-0,86$ $(p<0,01)$ ), este fato concorda com o proposto por Ricker (1979). O incremento observado nesta tabela entre as idades de 60 a 90 dias ocorreu em todas as famílias. Entre as idades de 90 a 150 dias, houve uma redução do igr em todas as famílias e pode ter sido em decorrência do tamanho corporal dos animais e o espaço disponível (tanque rede). Entre as idades de 150 a 210 dias, o igr aumentou em todos os grupos em função da transferência dos animais dos tanques redes para os viveiros. Em idades posteriores o crescimento na foi acentuado. 
Tabela 5. Média da taxa de crescimento instantâneo (igr (\%)), conforme as famílias de meios-irmãos paternos e idade.

\begin{tabular}{ccccccc}
\hline $\begin{array}{c}\mathbf{N}^{\mathbf{0}} \mathbf{d o} \\
\text { reprodutor }\end{array}$ & $\mathbf{6 0}$ & $\mathbf{9 0}$ & $\mathbf{1 5 0}$ & $\mathbf{2 1 0}$ & $\mathbf{2 7 0}$ & $\mathbf{3 3 0}$ \\
\cline { 2 - 6 } & $(1,05)$ & $7,02(8,62)$ & $1,74(24,55)$ & $1,40(57,02)$ & $1,19(116,35)$ & $0,56(162,52)$ \\
20 & $(1,46)$ & $5,06(6,66)$ & $1,76(19,19)$ & $1,78(55,69)$ & $1,09(106,88)$ & $0,73(165,27)$ \\
30 & $(1,59)$ & $4,91(6,95)$ & $1,69(19,22)$ & $2,01(64,18)$ & $0,79(103,34)$ & $0,84(170,80)$ \\
40 & $(1,64)$ & $4,75(6,81)$ & $1,59(17,69)$ & $1,99(58,42)$ & $1,15(116,84)$ & $0,75(183,46)$ \\
50 & $(1,09)$ & $5,92(6,45)$ & $1,57(16,56)$ & $2,48(73,53)$ & $0,83(121,14)$ & $0,65(178,83)$ \\
60 & $(1,52)$ & $4,82(6,45)$ & $1,70(17,90)$ & $2,10(63,05)$ & $1,22(131,04)$ & $0,58(186,18)$ \\
70 & $(1,88)$ & $4,88(7,94)$ & $0,84(13,14)$ & $2,22(49,72)$ & $1,13(97,71)$ & $0,88(158,30)$ \\
80 & $(2,20)$ & $4,93(9,66)$ & $0,81(15,70)$ & $2,04(53,43)$ & $0,92(93,03)$ & $0,64(136,84)$ \\
90 & $(1,01)$ & $6,28(6,64)$ & $1,86(20,24)$ & $2,15(73,73)$ & $0,52(100,54)$ & $0,76(158,94)$ \\
100 & $(1,76)$ & $4,90(7,66)$ & $1,81(22,76)$ & $1,83(68,57)$ & $0,85(114,33)$ & $0,61(165,09)$ \\
\hline
\end{tabular}

* Entre parênteses o peso médio em gramas.

Nos períodos de 90 a 150 dias e 150 a 210 dias, observou-se o aparecimento de "stanzas", conceito dado por Vasnetsov (1953) apud Ricker (1979), e que consiste em uma descontinuidade no crescimento. O aumento ou diminuição do igr (\%) pode revelar tais períodos. A herdabilidade para o crescimento apresenta um valor médio de 0,20, conforme Kinghorn (1983) e Gunnes e Gjedrem (1981). Desta forma, a seleção individual para essa característica pouco teria a acrescentar em ganho genético. No entanto, em se tratando de dados de progênie, a seleção pode ser eficiente em relação aos reprodutores, sendo este método eficiente para característica de baixa herdabilidade, conforme Kirpichnikov (1981).

\section{Conclusões}

Conforme os resultados obtidos nas condições em que o experimento foi realizado, conclui-se que: 1) o crescimento nas progênies de todos os reprodutores foi alométrico negativo, a exceção de um dos reprodutores que apresentou crescimento isométrico; 2) entre os valores determinados para o fator de condição de Fulton e o alométrico, este último mostrou-se mais eficiente na identificação dos reprodutores que apresentaram maior fator de condição através da progênie; 3) embora o fator de condição alométrico apresente herdabilidade baixa, o ganho genético pode ser incrementado através da seleção pela progênie.

\section{Referências}

BEACHAM, T. D.; MURRAY, C. B. A genetic analysis of body size in pink salmon (O. gorbusha). Genome, Ottawa, v. 30, n. 1, p. 31-35, 1988.

DOYON, J. F.; DOWNING, J. A.; MANIN, E. Variation in the condition of northern pike, Esox lucius. Canadian Journal of Fisheries and Aquatic Sciences, Ottawa, v. 45, n. 3, p. 479-483, 1988.

FISHER, S. J.; WILLIS, D. W.; POPE, K. L. Na assessment of burbot (Lota lota) Weight-length data juan North American populations. Canadian Journal of Zoology, Ottawa, v. 74, n. 3, p. 570-575, mar. 1996.

FRANK, K.; FOWLER, M. Fish conditions. Survey based in proceedings of the fisheries management studies Working group Bedford Institute of oceanography. Dartmouth, Nova Scotia, Canadá. Proceeding series, 15, 16 e 31 may 2001.82 p.

GALHARDO, E. Estudo genético do polimorfismo da transferrinas em populações selvagens e cultivadas de curimbatá, Prochilodus scrofa (Pisces, Prochilodontidae). 1989. Dissertação (Mestrado em Biologia) - Instituto de Biociências, Universidade de São Paulo, São Paulo.

GALL, G. A. E.; HUANG, N. Heritability and selection schemes for rainbow trout: body weigh. Aquaculture, Amsterdam, v. 73, n. 1-4, p. 43-56, 1988. 
GJERDE, B.; SCHAEFFER, L. R. Body traits in rainbow trout.II- Estimates of heritabilities and of phenotypic and genetic correlations. Aquaculture, Amsterdam, v. 80, n. 1-2, p. 25-44, 1989.

GUNNES, K.; GJEDREM, T. A genetic analysis of body weigth and length in rainbow trout reared in seawater for 18 months. Aquaculture, Amsterdam, v. 24, p. 161-174, 1981.

KANAZAWA, A.; TESHIMA, S. Microparticulate diets for fish larvae. In: SPARKS, A. K.(Ed.). New and innovative advances in biology/engineering with potential for use in aquaculture. Seattle: U.S. Department of Commerce, 1988. p.57-62. (NOAA Technical Report NMFS, 70)

KINGHORN, B. P. A review of quantitative genetics in fish breeding. Aquaculture, Amsterdam, v. 31, n. 2-4, p. 283-304, 1983.

KIRPICHNIKOV, V. S. Genetic bases of fish selection. Berlin: Springer Verlag, 1981.

LE CREN, E. D. The lenght-weight relationship and seasonal cycle in gonad weigth and condition in the perch (Perca fluviatis). Journal of Animal Ecology, Oxford, v. 20, n. 2, p. 201-219, 1951.

LEITE, R. G.; VERANI, J. R.; CESTAROLLI, M. A.; GODINHO, H. M.; FENERICH-VERANI, N.; BASILEMARTINS, M. A. Estudos biométricos do curimbatá, Prochilodus scrofa, em experimento de cultivo com suplementação alimentar-II. Crescimento. In: SIMPÓSIO BRASIlEIRO DE AQÜICULTURA, 3., 1984, São Carlos. Anais.... São Paulo: ABRAq, 1984. p. 345-365.

LLORET, J.; DE SOLA, L. G.; SOUPLET, A.; GALZIN, R. Effects of large-scale habitat variability on condition of demersal exploited fish in the North-Western Mediterranean. Journal of Marine Science, Dauphin Island, v. 59, n. 6, p. 1215-1227, 2002.

MAINARDES PINTO, C. S. R. M.; PAIVA, P.; ANTONIUTTI, D. M.; VERANI, J. R.; JUSTO, C. L. Influência do arraçoamento no crescimento do curimbatá, Prochilodus scrofa, em tanques experimentais de cultivo. In: SIMPÓSIO BRASILEIRO DE AQÜICULTURA, 3., 1984, São Carlos. Anais.... São Paulo: ABRAq, 1984. p. 313-327.

MCKAY, L. R.; IHSSEN, P. E.; FRIARS, G. W. Genetic parameters of growth in rainbow trout, Salmo gairdneri, prior to maturation. Canadian Journal of Genetics and Cytology, Ottawa, v. 28, n. 2, p. 306-312, 1986.
PADUA, H. B. Teores recomendados para manutenção de organismos aquáticos de água doce. Panorama da Aqüicultura, Rio de Janeiro, v. 4, n. 1, p. 8-9, 1994.

REFSTIE, T.; STEINE, T. A. Selection experiments with salmon. III-Genetic and environmental sources of atlhantic salmon in the fresh water phase. Aquaculture, Amsterdam, v. 14, n. 3, p. 221-234, 1978.

REFSTIE, T. Genetic and environmental sources of variation in body weight and length of rainbow trout fingerlings. Aquaculture, Amsterdam, v. 19, n. 4, p. 351$357,1980$.

RICKER, W. E. Growth rates and models. In: HOAR, W. S.; RANDALL, D. J.; BRETT, J. R. (Ed.). Fish physiology bioenergetics and growth. New York: Academic Press, 1979. v. 8, p. 677-743.

ROCHA, G. C.; BARROS, O. N. F.; GUIMARÃES, M. F. Distribuição espacial e características dos solos do campus da Universidade Estadual de Londrina-PR. Semina, Londrina, v. 12, n. 1, p. 25-27, 1991.

ROCHA, M. A. Estimativa de herdabilidade e correlações genéticas, fenotípicas e ambientais de características avaliadas no curimbatá (Prochilodus lineatus) em idades entre 60 a 330 dias. 1995. Tese (Doutorado em Aqüicultura) - Universidade Estadual Paulista, Jaboticabal.

ROCHA, M. A.; RIBEIRO, E. L. A. MIZUBUTI, I. Y.; SILVA, L. D. F. Estimativas de herdabilidade para o fator de condição alométrico em idades entre 90 a 330 dias no curimbatá (Prochilodus lineatus). Semina, Londrina, v. 21, n. 1, p. 41-44, 2000.

ROYCE, W. F. Introduction tothe fishery science. New York: Academic Press, 1972.

UNIVERSIDADE FEDERAL DE VIÇOSA. SAEG: manual de utilização do programa: sistema para análise estatística e genética. Viçosa: Imprensa Universitária, 1983.

SOKAL, R. R.; ROHLF, F. J. Introduccion a la bioestadistica. Barcelona: Editorial Reverté, 1980.

WELCH, H. E.; MILLS, K. H. Parking fish by scarring sofá fin rays. Canadian Journal of Fisheries and Aquatic Sciences, Ottawa, v. 38, n. 9, p. 1168-1170, 1981. 